\title{
DISTRIBUTION OF SELF-INCOMPATIBILITY ALLELES AND BREEDING STRUCTURE OF OPEN-POLLINATED CULTIVARS OF BRUSSELS SPROUTS
}

\author{
D. J. OCKENDON \\ Notional Vegetable Research Stotion, Wellesbourne, Worwick
}

Received 15.x.73

\begin{abstract}
SUMmary
Nineteen different $S$ alleles were found in 488 plants representing 16 cultivars of Brussels sprouts. Twelve of these $S$ alleles have previously been found in kale, and 7 are known only from Brussels sprouts. The number of $S$ alleles per cultivar varied from 4 to 13 , but for most cultivars the number fell in the range 7-10. Relatively unselected cultivars tend to have more $S$ alleles than highly selected ones. In every cultivar the $S$ allele frequencies are very unequal. The alleles with the greatest overall frequency are $S_{2}$ and $S_{5}$ and in almost all the cultivars tested one of these two alleles is the commonest. The rare $S$ alleles not only occur at very low frequencies, but also occur in few cultivars. The commonest $S$ alleles are recessive and the rare alleles are dominant. Dominant $S$ alleles normally confer a higher degree of self-incompatibility than recessive $S$ alleles. In Brussels sprouts cultivars a dynamic balance between outbreeding and inbreeding is achieved through the $S$ allele system. The recessive $S$ alleles, when present at high frequencies, allow some inbreeding, while the dominant $S$ alleles promote outbreeding even when they are present at low frequencies. Because dominant $S$ alleles confer high levels of self-incompatibility it is desirable to incorporate them into inbred lines used for the production of $F_{1}$ hybrid cultivars of Brussels sprouts. Such dominant alleles do occur in Brussels sprouts, but are rare and at least 20, and preferably 50, plants of a cultivar should be tested if rare $S$ alleles are being sought.
\end{abstract}

\section{INTRODUCTION}

$F_{1}$ HYBRID varieties of Brussels sprouts (Brassica oleracea var gemmifera) have become popular during the last 15 years because of their uniformity and high yield. A serious defect experienced with the majority of these varieties is the presence of sib plants (Johnson, 1972). The basis for the production of $F_{1}$ hybrid varieties of Brussels sprouts is the self-incompatibility of the inbred lines used. If the inbreds were completely self-incompatible, no sib seed would be produced. In most cases the inbreds used show some tendency towards self-compatibility under certain conditions (sometimes known as pseudo-compatibility). Self-incompatibility in Brassica oleracea is sporophytically determined and there is a multiple series of $S$ alleles (Thompson, 1957).

A high percentage of the inbred lines of Brussels sprouts currently available for making $\mathrm{F}_{1}$ hybrids contain the allele $S_{2}$ (Ockendon, 1972). Not only do most of these inbreds show a tendency towards self-compatibility, but also they cannot be crossed together readily because they all have the same $S$ allele. Thus there is a need for new inbreds which are more highly self-incompatible, and which have alleles other than $S_{2}$, so that the number of potential pair combinations of inbreds for $F_{1}$ hybrids can be greatly increased. 
An $S$ allele survey was undertaken to determine the total number of different $S$ alleles available in Brussels sprouts, to determine $S$ allele frequencies in different cultivars, and to search particularly for $S$ alleles which would be useful in new inbred lines. There is very little published information about the number and frequency of $S$ alleles in Brussels sprouts, but van Hal (Duiven) has collected a considerable amount of data on this topic, which has yet to be published.

\section{MAterials AND MEthods}

Nearly 500 plants representing 14 open-pollinated cultivars of Brussels sprouts were tested for their $S$ alleles. For two of these cultivars over a hundred plants of each were examined. The intention here was to detect alleles occurring at very low frequencies. One of the cultivars chosen (Darkmar 21) was thought to be relatively highly selected for agronomic characters, while the other (Select Giant) was thought to be less highly selected. For the other 12 cultivars between 12 and 25 plants were tested.

The plants tested were taken at random from each cultivar in order to give a picture of $S$ allele frequencies within a cultivar. The plants were not routinely checked for their self-incompatibility unless there was an indication that considerable self-compatibility might be present. Only 4 plants from the 14 cultivars recorded here showed marked self-compatibility and these were not allele tested because it is very difficult, if not impossible, to make reliable $S$ allele identifications in material which is markedly selfcompatible. In two other cultivars (Sanda and Northern Pride) many of the plants showed considerable amounts of self-compatibility. The results of allele testing plants of these two cultivars were incomplete and are not recorded here.

The $S$ allele tester lines used were those in the collection made at Cambridge by Thompson, which comprises 34 lines of kale (Brassica oleracea var acephala) and 3 lines of Brussels sprouts (B. oleracea var gemmifera). These have been given $S$ allele numbers 1-46. Four additional tester lines of Brussels sprouts were provided by van $\mathrm{Hal}$. These lines were given colour codes by van $\mathrm{Hal}$, but have been given numbers here, to accord with the numbering system started by Thompson. The numbers assigned are as follows :

$$
\begin{aligned}
& S_{60}=\text { Yellow } \\
& S_{61}=\text { Olive green } \\
& S_{62}=\text { Dark brown; } \\
& S_{63}=\text { Light blue. }
\end{aligned}
$$

Test pollinations were assessed as compatible or incompatible by examining pollen tube growth 24 hours after pollination. The pollen tubes were observed by the fluorescence technique described by Johnson (1971). Test crosses giving fewer than 20 pollen tubes at the top of the style were scored as incompatible and those giving more than 70 tubes were scored as compatible. The few tests which gave 20-70 pollen tubes were repeated and assessed as compatible or incompatible not only on the basis of the number of pollen tubes in the style but also according to the number of short inhibited pollen tubes found at the stigmatic surface. 
The $S$ allele tester plants were normally used as the male parents and two flowers were used in each test. Each test which gave an incompatible result was repeated and if any test gave a dubious result the fertility of both the male and female parents was checked. Pollen of the $S$ allele testers stored in gelatin capsules at $-20^{\circ} \mathrm{C}$. was frequently used in tests. The pollen viability of stored pollen was always checked whenever it was used.

\section{Results}

\section{(i) Total number of $\mathrm{S}$ alleles}

The results of the $S$ allele survey are summarised in table 1. The total number of $S$ alleles found in the plants tested was 19. Only one other $S$ allele was found in Brussels sprouts, namely $S_{11}$. Thirteen of the $S$ alleles found in Brussels sprouts also occur in kale, but the other 7 have been recorded only from Brussels sprouts. The total number of $S$ alleles found in kale is about 34 (Thompson, unpublished), of which 25 were found in a single cultivar. As there is a greater range of morphological type in kale than in Brussels sprouts, it is noteworthy that there are more $S$ alleles in the former than in the latter.

The total number of $S$ alleles found in any one cultivar ranges from a maximum of 13 in Select Giant to 4 in Vremo, but the majority of cultivars had between 7 and $10 S$ alleles. Apart from Select Giant and Darkmar, similar numbers of plants of each cultivar were tested. Market Gem and Vremo have markedly fewer $S$ alleles than the other cultivars tested, and the possible significance of this will be discussed later. Select Giant contained 5 more $S$ alleles than Darkmar 21, although almost equal numbers of plants of each were tested. This result has fulfilled the expectation that an old, relatively unselected cultivar would contain more $S$ alleles than a newer, highly selected cultivar.

\section{(ii) $\mathrm{S}$ allele frequencies}

The overall $S$ allele frequencies are given in table 2. The commonest $S$ alleles were $S_{2}$ and $S_{5}$, and these were considerably commoner than any of the others. The overall $S$ allele frequencies give a reasonable picture of the situation in Brussels sprouts as a whole, but the precise figures depend on the particular cultivars tested. Thus the figures for $S_{60}, S_{61}$ and $S_{62}$ are probably underestimates, and would have been greater if more Dutch material had been tested.

A similar survey of $S$ alleles in Brussels sprouts was made by van $\mathrm{Hal}$ (unpublished). He deliberately used material selected for agronomic characters and discarded plants which showed any appreciable selfcompatibility. Nevertheless, the general picture found by him was similar to that presented here. Differences between the two sets of data can largely be accounted for by the fact that van Hal worked chiefly with Dutch cultivars whereas the work described here was chiefly with English cultivars.

The general pattern of $S$ allele distribution was much the same in each of the cultivars examined. In most cases there was a single allele either $S_{2}$ or $S_{5}$ which was appreciably more common than any of the other alleles found in the cultivar and had a frequency of about 30-60 per cent. In a few cases $S_{2}$ and $S_{5}$ had a very high and nearly equal frequency (e.g. in 
TABLE 2

Relative overall $\mathrm{S}$ allele frequencies in 488 plants representing 14 cultivars of Brussels sprouts

$\begin{array}{lcc} & \begin{array}{c}\text { Number of times } \\ \text { found }\end{array} & \text { Frequency }(\%) \\ S_{2} & 183 & 18 \cdot 75 \\ S_{5} & 145 & 14 \cdot 86 \\ S_{45} & 78 & 7 \cdot 99 \\ S_{46} & 66 & 6 \cdot 76 \\ S_{14} & 62 & 6 \cdot 35 \\ S_{13} & 46 & 4 \cdot 71 \\ S_{29} & 42 & 4 \cdot 30 \\ S_{23} & 40 & 4 \cdot 10 \\ S_{15} & 37 & 3 \cdot 79 \\ S_{63} & 33 & 3 \cdot 38 \\ S_{39} & 28 & 2 \cdot 87 \\ S_{7} & 16 & 1 \cdot 64 \\ S_{27} & 9 & 0 \cdot 92 \\ S_{60} & 6 & 0 \cdot 61 \\ S_{61} & 6 & 0 \cdot 61 \\ S_{36} & 5 & 0 \cdot 51 \\ S_{62} & 5 & 0 \cdot 51 \\ S_{35} & 2 & 0 \cdot 20 \\ S_{22} & 1 & 0 \cdot 10 \\ \text { Unknown } & 166 & 17 \cdot 01\end{array}$

cvs. Rous Lench and Gibsons). $S_{2}$ was a little more common overall than $S_{5}$ and was the only allele which was found in every one of the 14 cultivars examined.

The second category of $S$ alleles comprised those which were moderately common in that they occurred in at least half of the cultivars tested, and typically had frequencies of 10-30 per cent. This category comprises $S_{13}$, $S_{14}, S_{15}, S_{45}$ and $S_{46}$. The third category comprises the rare and very rare alleles which were found in less than half the cultivars tested and usually had frequencies of less than 20 per cent. This group consists of $S_{7}, S_{22}, S_{23}$, $S_{27}, S_{29}, S_{35}, S_{36}, S_{39}, S_{60}, S_{61}, S_{62}, S_{63}$.

\section{(iii) $\mathrm{S}$ allele distribution in different types of cultivar}

As cultivars with a common origin might be expected to have the same $S$ alleles, it is worth asking if there are any differences in the pattern of $S$ allele distribution in different types of cultivar. As it is very difficult to classify Brussels sprout cultivars on morphological grounds, the most useful way of grouping cultivars is according to their area of origin. Three types of cultivar have been recognised here, namely Bedfordshire Types, Old English Types and Dutch Types. The first of these is probably more homogeneous than the other two. Differences in the $S$ alleles found in these types are apparent from table 1. The English Types (Bedfordshire and Old English) have $S_{22}, S_{23}, S_{27}, S_{35}, S_{36}, S_{39}$ and $S_{63}$ which are lacking in the Dutch Types tested, while the latter have $S_{61}$ and $S_{62}$ which are lacking in the English Types. Furthermore, $S_{5}$ which is common in all the English Types except one, is present in only one Dutch Type. Differences between the Bedfordshire Types and Old English Types are less marked than those between English and Dutch Types. $S_{7}, S_{22}, S_{35}, S_{60}$ and $S_{63}$, which occur in the Old English Types, are lacking in the Bedfordshire Types. The 
differences mentioned should not be taken as absolute, because if more Dutch cultivars had been tested they might have been found to contain other $S$ alleles. Nevertheless, important differences between types of cultivar can be found in respect of their $S$ alleles. As expected, cultivars which are assumed to have a common origin are similar in their $S$ allele constitutions.

\section{Discussion}

The results show very clearly that $S_{2}$ is the commonest allele in Brussels sprouts, both in so far as it is the only allele which was found in every cultivar tested, and in so far as it has the highest overall frequency, being found in almost 38 per cent of the plants tested. Similar results were obtained by Thompson and Taylor (1966) who found $S_{2}$ in all seven of the selfincompatible cultivated forms of Brassica oleracea which they tested. The overall frequency of $S_{2}$ in their material was 48 per cent, but varied from 10 per cent in curly kale to 76 per cent in kohl rabi. The high frequency of $S_{2}$ (and to a lesser extent of $S_{5}$ ) in Brussels sprouts helps to explain why so many of the inbreds currently available contain these two alleles. Despite the high frequency of these two alleles there are at least 10 other $S$ alleles in Brussels sprouts which are not too difficult to find and which should give better levels of self-incompatibility than $S_{2}$ and $S_{5}$. The very high frequency of $S_{2}$ in Brussels sprouts has been something of a puzzle because one might expect that natural selection would tend to maintain all the $S$ alleles in a population at about the same frequency, because of selection in favour of rare $S$ alleles and against common $S$ alleles (Wright, 1939). One possibility is that $S_{2}$ is closely linked to a character of agronomic importance which has been selected for. Another possibility of great interest is that the high frequency of $S_{2}$ is linked with the fact that it is a recessive allele. This will be discussed in more detail later.

One aim of this work was to establish how many plants of a cultivar ought to be tested to detect all the $S$ alleles present and to obtain reasonable estimates of their frequencies. If $n$ is the number of plants sampled and $p$ is the frequency of a given allele per plant, the chance of finding that allele is given by the expression $1-(1-p) n$. Thus one has a 97 per cent chance of finding an allele occurring with a frequency of 20 per cent if 15 plants are examined. To be sure of finding all the rarer alleles, many more plants must be examined. Fifty plants are required to give a 95 per cent chance of detecting an allele with a frequency of 6 per cent and nearly 200 are required if the frequency is only 2 per cent. In practice, the first 16 plants of Select Giant which were tested, contained 9 different $S$ alleles and only $13 S$ alleles were found in the total of 133 plants of this cultivar which were examined. In order to be sure of detecting the majority of $S$ alleles in a cultivar it would seem wise to test at least 20 plants. Testing 50 plants gives a good chance of detecting most of the rare alleles which have a frequency of 2 per cent or more. Alleles with even lower frequencies do occur (e.g. $S_{22}$ and $S_{35}$, table 1), but they are likely to be too few in number to be worth searching for unless these particular $S$ alleles are especially wanted. However, the greater the number of plants tested, the more accurate the estimates of allele frequencies. Preliminary screening of material to eliminate plants which show any appreciable self-compatibility, 
as was done by van Hal, should facilitate the search for rare alleles in particular.

As will be seen from table 1 , about $30-40$ per cent of the plants in most cultivars contained one $S$ allele which remains unidentified. Possible reasons for this are as follows:

(i) Errors in testing procedure and inability to test every plant with every tester line.

(ii) The plants contain $S$ alleles which are not present in the tester collection (i.e. new $S$ alleles).

(iii) The plants are homozygous for their $S$ allele.

(iv) The plants contain a weak $S$ allele which was not detected because of dominance effects.

The plants in which only one allele could be found have been kept and will be tested further. The first possibility is not likely to apply except in very few cases. The other three possibilities are being investigated by selfing the plants concerned and testing the progeny against the allele which is known to be present. If all the progeny are incompatible with the known tester then homozygosity is demonstrated. If not, then the plants which are compatible with the tester must be homozygous for the unknown allele. Once the unknown allele has been isolated it can be tested against the whole $S$ allele collection, and if it proves to be unidentifiable, it is regarded as a new allele and will eventually be added to the $S$ allele collection. If the unknown allele proves to be identifiable once isolated, but was unidentifiable in the heterozygous condition in the original plant, this is a strong indication that it was masked by a dominant allele.

Dominance between $S$ alleles in Brassica oleracea has been well established both in kale (Thompson, 1968; Thompson and Taylor, 1971) and in Brussels sprouts (Haruta, 1962; van Hal, unpublished). Dominance relationships are not linear in most cases, and partial dominance appears to be more common than complete dominance. Dominance is expressed more clearly in the pollen than in the stigma, and the plants to be tested were used as the female parents because the activity of a recessive $S$ allele is much less likely to be completely masked in the stigma than in the pollen. The 3 most recessive alleles found by Thompson (1968) were $S_{2}, S_{5}$ and $S_{15}$. It is very significant that the two most common $S$ alleles in Brussels sprouts, are two of these recessive alleles $\left(S_{2}\right.$ and $\left.S_{5}\right)$ and that $S_{15}$ is quite common. Thompson and Taylor (1971) have shown that partial selfcompatibility in kale can be attributed to the presence of recessive alleles. Several inbred lines of Brussels sprouts which carry $S_{2}$ or $S_{5}$ are partially self-compatible (Ockendon, unpublished).

The possibility that some of the plants tested may be homozygous for their $S$ alleles will now be considered. Homozygotes can arise from selfcompatibility and although low levels of self-compatibility were found in many of the plants tested, only 4 showed high self-compatibility. $S$ allele homozygotes can also arise because of dominance between $S$ alleles (table 3 ), and this may be a more important mode of origin in those cultivars with little self-compatibility, as all cultivars tested have high frequencies of recessive $S$ alleles. Plants with an $S$ allele in common are expected to be cross-incompatible unless that allele is recessive, in which case it will be $33 / 2-I$ 
TABLE 3

Origin of plants homozygous for their $\mathrm{S}$ alleles. Cross-compatibilities are indicated for the six possible heterozygotes in a population containing the recessive alleles $\mathrm{S}_{2}$ and $\mathrm{S}_{5}$ and the dominant alleles $\mathrm{S}_{29}$ and $\mathrm{S}_{39}$

\begin{tabular}{|c|c|c|c|c|c|c|}
\hline 오 후 & $\dot{S}_{2} S_{5}$ & $S_{2} \dot{S}_{29}$ & $S_{5} \dot{S}_{29}$ & $S_{2} \dot{S}_{39}$ & $S_{5} \dot{S}_{39}$ & $\dot{S}_{29} \dot{S}_{39}$ \\
\hline$\dot{S_{2}} \dot{S_{5}}$ & & $\begin{array}{c}(+) \\
S_{2} S_{2}\end{array}$ & $\begin{array}{c}(+) \\
S_{5} S_{5}\end{array}$ & $\begin{array}{c}(+) \\
S_{2} S_{2}\end{array}$ & $\begin{array}{l}(+) \\
S_{5} S_{5}\end{array}$ & + \\
\hline$\dot{S_{2}} \dot{S_{29}}$ & - & & - & $\begin{array}{c}(+) \\
S_{2} S_{2}\end{array}$ & + & - \\
\hline$\dot{S_{5}} \dot{S_{29}}$ & $\begin{array}{c}(+) \\
S_{5} S_{5}\end{array}$ & - & & + & $\begin{array}{c}(+) \\
S_{5} S_{5}\end{array}$ & - \\
\hline$\dot{S_{2}} \dot{S_{39}}$ & - & $\begin{array}{c}(+) \\
S_{2} S_{2}\end{array}$ & + & & - & - \\
\hline$S_{5} \dot{S_{39}}$ & $\begin{array}{c}(+) \\
S_{5} S_{5}\end{array}$ & + & $\begin{array}{c}(+) \\
S_{5} S_{5}\end{array}$ & - & & - \\
\hline$\dot{S}_{29} \dot{S}_{39}$ & + & - & - & - & - & \\
\hline
\end{tabular}

- Indicates that the allele is active (dominant). Non-active (recessive) alleles are not marked.

+ Compatible combinations with no $S$ allele common to both parents.

(+) Compatible combinations with a common $S$ allele.

- Incompatible combinations.

Only homozygous offspring are indicated. Although plants homozygous for the recessive alleles may occur, plants homozygous for the dominant alleles will not occur in this system.

masked by any dominant allele which is present. This partial crosscompatibility between plants carrying the same recessive $S$ allele gives rise to plants which are homozygous for recessive $S$ alleles, but would not be expected to give plants homozygous for dominant $S$ alleles. It is likely that some of the plants found to contain recessive $S$ alleles by Thompson and Taylor (1966, table 1) were in fact homozygous for their $S$ alleles.

It is pertinent to consider the number of $S$ alleles necessary to maintain a population with sporophytic incompatibility. Assuming no self-compatibility and no dominance, at least $4 S$ alleles are necessary to maintain the population. With this number of $S$ alleles only 20 per cent of the possible crosscombinations are compatible, if each $S$ allele has the same frequency. The percentage of compatible combinations rises sharply at first, as each new $S$ allele is introduced (table 4 ), but when there are 12 alleles in the population, there is 69 per cent cross-compatibility and addition of each further $S$ allele gives only a small increase in this percentage. It is noteworthy that none of the cultivars tested had fewer than $4 S$ alleles, and that all except two had at least $7 S$ alleles which would give an estimated cross-compatibility of 50 per cent. The cultivar Vremo with only $4 S$ alleles also has very unequal $S$ allele frequencies, so that the cross-compatibility would be expected to be well below 20 per cent, were it not for the presence of recessive $S$ alleles. 
It will now be argued that as a direct result of single plant selection and hence declining numbers of $S$ alleles in open-pollinated cultivars, there has been strong selection in favour of partial self-compatibility and dominance interactions between $S$ alleles. This view was taken by Thompson and Taylor (1966) who also suggested that more intense selection has probably reduced the number of $S$ alleles in the horticultural types of Brassica oleracea compared with the agricultural types such as marrow-stem kale. New $S$ alleles can be introduced into a cultivar only by hybridisation with another cultivar, as the effect of mutation in generating new $S$ alleles is so small as to be negligible (Pandey, 1972). In contrast, loss of $S$ alleles from a cultivar can occur very easily whenever a small number of plants is selected to perpetuate a cultivar. Loss of rare $S$ alleles is particularly likely to occur.

\section{TABLE 4}

Theoretical percentages of compatible cross-pollinations in populations with different numbers of $\mathrm{S}$ alleles and sporophytic self-incompatibility. It is assumed that each $\mathrm{S}$ allele has the same frequency and that the recessive alleles are completely recessive

\begin{tabular}{|c|c|c|c|}
\hline \multirow{2}{*}{ No. $S$ alleles } & \multicolumn{3}{|c|}{ Percentage of compatible cross-combinations } \\
\hline & $\begin{array}{l}\text { (a) No recessive } \\
\text { alleles }\end{array}$ & $\begin{array}{l}\text { (b) One recessive } \\
\text { allele }\end{array}$ & (c) Two recessive \\
\hline $\begin{array}{l}\text { No. } S \text { alleles } \\
4\end{array}$ & 20 & 40 & $\begin{array}{l}\text { alleles } \\
60\end{array}$ \\
\hline 5 & 33 & 47 & 60 \\
\hline 6 & 43 & 52 & 62 \\
\hline 7 & 50 & 57 & 64 \\
\hline 8 & 56 & 61 & 67 \\
\hline 9 & 60 & 64 & 69 \\
\hline 10 & 64 & 67 & 71 \\
\hline 11 & 67 & 70 & 73 \\
\hline 12 & 69 & 72 & 74 \\
\hline 13 & 71 & & \\
\hline 14 & 73 & & \\
\hline 15 & 75 & & \\
\hline 20 & 81 & & \\
\hline 30 & 87 & & \\
\hline 50 & 92 & & \\
\hline 100 & 96 & & \\
\hline
\end{tabular}

Cultivars with $4 S$ alleles or fewer are likely to fail because of low seed yields if they do not have appreciable self-compatibility or dominance between $S$ alleles. Both of these features are found in Brussels sprouts but they may not be of equal importance in the breeding system of this crop. Self-compatibility is essentially an inbreeding mechanism, but a system based on dominance between $S$ alleles still allows a large amount of outbreeding by increasing the cross-compatibility of combinations which share a common $S$ allele (table 3 ). The extent to which recessive $S$ alleles increase the amount of cross-compatibility is shown in table 4 . In a population with $4 S$ alleles the amount of cross-compatibility is doubled if one of these alleles is recessive and is increased three-fold if two of the alleles are recessive. When $12 S$ alleles are present, the effect of the recessive $S$ alleles appears to be very small, but the figures given are an underestimate because they were calculated on the basis that each $S$ allele had the same frequency in the population whereas in most cases the recessive alleles are more 
frequent than the dominant ones. Nevertheless, the fewer the number of $S$ alleles in a population, the greater the effect of the recessive alleles is likely to be.

A comparison between the breeding systems of Brussels sprouts and cauliflowers is instructive as both crops have been subject to intense selection for uniformity. The breeding system of cauliflowers has been studied in detail by Watts (1963). He found that summer cauliflowers are completely self-compatible, autumn cauliflowers are partially self-incompatible and winter cauliflowers even more self-incompatible but not completely so. The summer types show no inbreeding depression, but this does occur to varying degrees in the autumn and winter types. The summer cauliflowers are considered to be derived from the autumn and winter types. In the present study, cultivars of Brussels sprouts with a considerable proportion of self-compatible plants were intentionally discarded. These cultivars are in fact less numerous than those in which only a few plants are self-compatible and apparently no Brussels sprout cultivar consists entirely of self-compatible individuals. Repeated selfing appears to be invariably associated with inbreeding depression in Brussels sprouts. The extent of this depression varies widely. Some inbreds are lost altogether during the course of an inbreeding programme. Others are comparatively vigorous, but still have yields considerably lower than the $F_{1}$ hybrids which can be produced from them. Although self-compatibility exists in some cultivars of Brussels sprouts, none is as self-compatible as summer cauliflowers. This is associated with the fact that inbreeding depression occurs in the former, but not in the latter.

In highly selected open-pollinated cultivars of Brussels sprouts two opposing tendencies are postulated. On the one hand, strong selection for uniformity favours weak $S$ alleles and subsequent inbreeding, and on the other hand, selection for vigour and high yield favours strong $S$ alleles and subsequent outbreeding. A dynamic balance between these two trends is achieved by means of the recessive $S$ alleles. A plant with two recessive $S$ alleles is seldom completely self-compatible, but may be sufficiently self-compatible to give some seed set in the absence of compatible crosspollen. A plant with one recessive and one dominant $S$ allele is likely to be highly self-incompatible, but will be effectively cross-compatible with any plant not carrying the same dominant allele.

The hypothesis just proposed for the breeding structure of open pollinated varieties of Brussels sprouts indicates a balance between outbreeding and inbreeding, depending partly on the number of $S$ alleles present in the population. The unequal allele frequencies become intelligible in view of the fact that the most common alleles are the recessive ones. When the number of $S$ alleles in the population is low, there will be increasing selection in favour of the recessive alleles in order to give sufficient self- and crosscompatibility to ensure adequate seed set. This tendency is balanced by selection in favour of retaining at least a few dominant $S$ alleles to promote outbreeding. The dominant $S$ alleles can have their effect at low frequencies, whereas the recessive alleles must be more frequent in order to be effective. The high frequencies of the recessive $S$ alleles will be maintained, if not increased, because of the greater cross-compatibility of the plants carrying the recessive $S$ alleles (table 3 ). This explanation agrees with the finding that in the majority of cultivars examined, it is always one of two alleles 
which is commonest $\left(S_{2}\right.$ and $\left.S_{5}\right)$. If the unequal allele frequencies were due to random effects produced by artificial selection, there is no reason why the commonest allele in one cultivar should also be very common in other cultivars.

There has been some work and considerable speculation about the number of different $S$ alleles present in self-incompatible species. Williams (1947) found $41 S$ alleles in 24 plants of one cultivar of red clover (Trifolium pratense) and $37 S$ alleles in 20 plants of another cultivar of that species. Emerson (1939) found $37 S$ alleles (later 45 (Wright, 1964)) in a population of Oenothera organensis estimated by him to contain less than 500 plants. Sampson (1967) studied the occurrence of 9 different $S$ alleles in 45 plants of Raphanus raphanistrum representing 5 different wild populations. From his results he calculated that the total number of $S$ alleles in this species is probably between 25 and 34 . Bateman (1954) studied a wild population of Iberis amara. Fifty-two plants were tested, 5 of which were self-compatible, and the total number of $S$ alleles in the population estimated to be at least 22. Bateman (1947) made some calculations on the data of Williams (1947) and estimated that one cultivar of red clover contained a total of 171 $S$ alleles and the other cultivar contained a total of $308 S$ alleles. These figures may not be particularly accurate estimates, but are examples of the very large numbers of $S$ alleles which are thought to occur in a single species or even in a single population. There has been some difficulty in explaining the occurrence of such large numbers of $S$ alleles in view of known mutation rates (Pandey, 1972).

The number of $S$ alleles present in kale and Brussels sprouts has been fairly firmly established, not only because of the very large number of plants tested, but because of the existence of the Brassica $S$ allele tester collection which enables the $S$ allele constitution of individual plants to be determined. Allowing for some duplications and one or two uncertainties, a total of 41 different $S$ alleles has been established, of which 13 are common to both kale and Brussels sprouts. It is very doubtful if more than 50 different $S$ alleles are present in kale and Brussels sprouts. Cabbages have not been so thoroughly tested, but they are unlikely to contain more than $10 S$ alleles not already found in kale and Brussels sprouts. Other cultivated forms of Brassica oleracea might contain a few more $S$ alleles, but preliminary tests suggest that the commonest alleles in kale and Brussels sprouts are also the commonest in most of the other cultivated forms (Ockendon, unpublished; Thompson, 1968). Thus it is unlikely that more than 70 different $S$ alleles occur in all the cultivated forms of Brassica oleracea. The occurrence of further $S$ alleles in wild forms of Brassica oleracea is a matter for speculation.

The number of $S$ alleles found in Brassica oleracea (41) is comparable with that for Iberis amara (22) and Raphanus raphanistrum (25-34), but is very much less than the number estimated for Trifolium pratense (171-308), although the number actually found in Trifolium pratense (41) is the same as that in Brassica oleracea. It is possible that Cruciferous species such as Brassica oleracea, Iberis amara and Raphanus raphanistrum with sporophytic selfincompatibility and marked dominance between $S$ alleles in the pollen have appreciably fewer $S$ alleles than species such as Oenothera organensis and Trifolium pratense which have a gametophytic system and consequently no possibility of $S$ allele interaction in the pollen. 


\section{Conclusion}

It is widely accepted that a thorough understanding of the breeding system of a crop is essential background information for a breeding programme. What is not so widely recognised is that there may be subtle differences in breeding systems between closely related crops, and even between cultivars of a single crop. Direct experimental measurement of the percentage of outcrossing in any one cultivar is rather a lengthy procedure. Study of the $S$ allele system in Brassica oleracea provides very useful indirect evidence about breeding systems. Knowledge of $S$ allele frequencies and dominance relationships provides a simple way of comparing the breeding behaviour of different cultivars. A cultivar with only $4 S$ alleles, 3 of which are recessive, will certainly be subject to more inbreeding than a cultivar having $11 S$ alleles, 4 of which are recessive. The recessive $S$ alleles play a crucial role in allowing partial inbreeding, without undermining the integrity of the self-incompatibility system as a whole.

Information about the relative frequencies of $S$ alleles in Brussels sprouts as a whole and of the distribution of particular $S$ alleles in certain types of cultivar is a useful background for the routine identification of $S$ alleles in inbred lines. The amount of labour involved in the testing procedure can be reduced if the commonest alleles are tested for first.

The results given here are valuable not only in the breeding of $F_{1}$ hybrid varieties of Brussels sprouts, but also in the breeding of other types of cultivar. Dominant $S$ alleles do occur in Brussels sprouts, but they occur at low frequencies, and there are very few in any one cultivar (1-5 for the cultivars studied here). Dominant $S$ alleles are probably more easily found in the older, less intensely selected varieties than in the newer, highly selected varieties. There are good arguments for trying to produce new inbred lines with dominant $S$ alleles. These will be more highly self-incompatible than most existing inbreds, and cross-compatible with the majority of them. If one is thinking in terms of double-cross hybrids or synthetics, a range of inbreds carrying some of the rarer $S$ alleles is highly desirable because of the limited number of $S$ alleles currently available in inbreds. As far as synthetics are concerned, it is highly desirable that at least 4 different $S$ alleles should be present, and it is probably best if the most recessive $S$ alleles are excluded. This study has been useful not only in finding material which can be utilised in the production of new inbred lines, but also in providing a basis for the rational selection of material for a breeding programme, whatever type of cultivar is being sought.

Acknowledgments.- Thanks are due to Dr K. F. Thompson (Cambridge) and Mr J. G. van Hal (Duiven) for kindly providing $S$ allele tester material, to Mrs J. Bond and Mrs L. Currah for their excellent technical assistance and to my colleagues for helpful comment and criticism.

\section{References}

Bateman, A. J. 1947. Number of $S$ alleles in a population. Nature, 160, 337.

BAtEMAN, A. J. 1954. Self-incompatibility systems in Angiosperms. II. Iberis amara. Heredity, 8, 305-332.

EMERson, s. 1939. A preliminary survey of the Oenothera organensis population. Genetics, 24, 524-537. 
HARUTA, T. 1962. Studies on the genetics of self-and cross-incompatibility in cruciferous vegetables. Res. Bull. No. 2. Takii Pl. Breed. Exp. Stn., Kyoto, Japan.

Johnson, A. G. 1971. Factors affecting the degree of self-incompatibility in inbred lines of Brussels sprouts. Euphytica, 20,561-573.

Johnson, A. G. 1972. Problems in breeding and seed production of hybrid sprouts. Comml. Grow., No. 4012, pp. 749-750.

ockendon, D. J. 1972. Incompatibility studies. National Vegetable Research Station Ann. Rep. for 1971, pp. 28-29.

PANDEY, K. K. 1972. Origin of genetic variation: regulation of genetic recombination in the higher organisms-a theory. Theoret. Appl. Genetics, 42, 250-261.

SAMPSON, D. R. 1967. Frequency and distribution of self-incompatibility alleles in Raphanus raphanistrum. Genetics, 56, 241-251.

THомPson, K. F. 1957. Self-incompatibility in marrow-stem kale, Brassica oleracea var acephala. I. Demonstration of a sporophytic system. F. Genetics, 55, 45-60.

тHомson, к. F. 1968. Classified $S$ alleles for Brassica breeders. In Brassica meeting of Eucarpia (G. E. Dixon, ed.), pp. 25-28.

THOMSON, K. P., AND TAYLOR, J. P. 1966. The breakdown of self-incompatibility in cultivars of Brassica oleracea. Heredity, 21, 637-648.

THOMPSON, K. F., AND TAYLOR, J. P. 1971. Self-compatibility in kale. Heredity, 27, 459-471.

WATTS, L. E. 1963. Investigation into the breeding system of cauliflower Brassica oleracea var botrytis L. I. Studies of self-incompatibility. Euphytica, 12, 323-340.

williams, w. 1947. Genetics of red clover (Trifolium pratense L) compatibility. III. The frequency of incompatibility $S$ alleles in two non-pedigree populations of red clover. 7. Genetics, $48,69-79$.

WRIGHT, s. 1939. The distribution of self-sterility alleles in populations. Genetics, 24, 538552.

WRIGHT, s. 1964. The distribution of self-sterility alleles in populations. Evolution, 18, 609-619. 